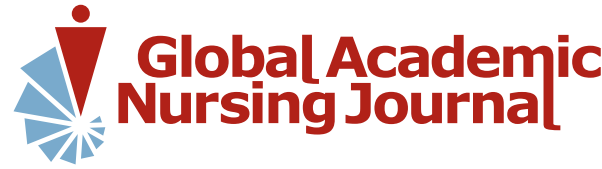

\section{O tratamento diretamente observado para tuberculose pode ser substituído e, ainda assim, obter sucesso no tratamento?}

Can the directly observed treatment for tuberculosis be replaced and still be successful in treatment?

¿Se puede reemplazar el tratamiento directamente observado para la tuberculosis y aún así tener éxito en el tratamiento?

\author{
Eunice Maria Pereira Côrtes ${ }^{1}$ \\ ORCID: 0000-0002-0353-4257 \\ Wenderson Bruno Herculano da \\ Silva ${ }^{1}$ \\ ORCID: 0000-0001-5288-4264 \\ Michelle Amorim Ferreira ${ }^{1}$ \\ ORCID: 0000-0001-5585-4925 \\ Carolini Moreira Mattos ${ }^{1}$ \\ ORCID: 0000-0002-3139-9419 \\ Paulo Roberto Ferreira \\ Machado ${ }^{1}$ \\ ORCID: 0000-0003-3578-6907 \\ Tatiana Araújo de Lima ${ }^{1}$ \\ ORCID: 0000-0001-8930-0672 \\ Cristiano Bertolossi Marta ${ }^{1}$ \\ ORCID: 0000-0002-0635-7970 \\ Milton Domingues da Silva \\ Junior $^{1}$ \\ ORCID: 0000-0003-1163-9005
}

${ }^{1}$ Universidade Veiga de Almeida. Rio de Janeiro, Brasil.

\section{Como citar este artigo:}

Côrtes EMP, Silva WBH, Ferreira MA, Mattos CM, Machado PRF, Lima TA, Marta CB, Silva Junior MD. O tratamento diretamente observado para tuberculose pode ser substituído e, ainda assim, obter sucesso no tratamento? Glob Acad Nurs. 2021;2(1):e74.

https://dx.doi.org/10.5935/26755602.20200074

Autor correspondente:

Eunice Maria Pereira Côrtes

E-mail: eunicempc@gmail.com

Editor Chefe: Caroliny dos Santos Guimarães da Fonseca

Editor Executivo: Kátia dos Santos Armada de Oliveira

Submissão: 12-01-2021

Aprovação: 18-01-2021

\section{Resumo}

O objetivo deste estudo foi verificar a efetividade da aplicação do telemonitoramento para o acompanhamento do tratamento dos pacientes com TB, segundo a visão do enfermeiro. Foi realizado um estudo qualiquantitativo desenvolvido em uma UBS alocada na Zona Norte do Rio de Janeiro onde 10 profissionais (entre enfermeiros e residentes de enfermagem no curso de seu segundo ano de residência profissional) avaliaram os tratamentos para TB em curso durante a pandemia. Foi aplicada a análise de conteúdo de Bardin neste estudo. Houveram percepções distintas sobre esta ferramenta utilizada para o a acompanhamento desses pacientes. Foi possível perceber que o telemonitoramento pode ser aplicado como aliado para acompanhamento de pacientes em tratamento de doenças crônicas, sobretudo aqueles que possuem dificuldades de acesso ao atendimento presencial, embora deva-se citar que há certa perda de qualidade no atendimento pois esse método não possibilita observar informações que não sejam verbalizadas pelo paciente.

Descritores: Atenção Básica; COVID-19; Pandemias; Telemonitoramento; Tuberculose.

\begin{abstract}
The aim of this study was to verify the effectiveness of the application of telemonitoring for monitoring the treatment of patients with TB, according to the nurse's view. A qualitative and quantitative study was carried out in a UBS located in the North Zone of Rio de Janeiro, where 10 professionals (including nurses and nursing residents in their second year of professional residency) evaluated TB treatments underway during the pandemic. Bardin's content analysis was applied in this study. There were different perceptions about this tool used to monitor these patients. It was possible to realize that telemonitoring can be applied as an ally for monitoring patients undergoing treatment for chronic diseases, especially those who have difficulties accessing face-to-face care, although it should be mentioned that there is a certain loss of quality in care because this method does not allow observe information that is not verbalized by the patient.
\end{abstract}

Descriptors: Primary Attention; COVID-19; Pandemic; Telemonitoring; Tuberculosis.

\section{Resumén}

El objetivo de este estudio fue verificar la efectividad de la aplicación de la telemonitorización para el seguimiento del tratamiento de los pacientes con TB, según la opinión de la enfermera. Se realizó un estudio cualitativo y cuantitativo en una UBS ubicada en la Zona Norte de Río de Janeiro, donde 10 profesionales (entre enfermeras y residentes de enfermería en el transcurso de su segundo año de residencia profesional) evaluaron los tratamientos de TB en curso durante la pandemia. En este estudio se aplicó el análisis de contenido de Bardin. Hubo diferentes percepciones sobre esta herramienta utilizada para monitorear a estos pacientes. Se pudo constatar que la telemonitorización se puede aplicar como un aliado para el seguimiento de los pacientes en tratamiento por enfermedades crónicas, especialmente aquellos que tienen dificultades para acceder a la atención presencial, aunque cabe mencionar que existe una cierta pérdida de calidad en la atención. porque este método no permite observar información que no sea verbalizada por el paciente.

Descriptores: Atención Básica; COVID-19; Pandemias; Telemonitorización; Tuberculosis. 


\section{Introdução}

A enfermidade causada, principalmente, pelo Mycobacterium tuberculosis (também chamado bacilo de Koch) ocupa o lugar de doença infectocontagiosa com a maior taxa de mortalidade no mundo, conforme a Organização Mundial de Saúde (OMS), superando, inclusive, a Aids ${ }^{1}$.

Sua apresentação mais comum é a pulmonar e a contaminação ocorre pela inalação de bacilos lançados no ar através de tosse, espirro e fala da pessoa doente com Tuberculose (TB) ativa pulmonar ou laríngea. Há uma estimativa do Ministério da Saúde (MS) que um paciente infectado pode contaminar de 10 a 15 pessoas durante um ano ${ }^{1,2}$.

O tratamento ocorre através de esquemas de antibióticos combinados e prescritos de acordo com o metabolismo bacilar. Tem duração mínima de seis meses com uso diário da antibioticoterapia definida para cada caso. Em geral, depois de 15 dias de tratamento o indivíduo não é mais bacilífero, o que significa que não é mais capaz de transmitir a doença².

A OMS define a Atenção Primária à Saúde como se tratando do primeiro nível de acesso ao sistema de saúde e ainda que cuida de pessoas, observando os indivíduos de maneira holística e não agindo somente em busca da cura e/ou tratamento da enfermidade, mas atuando em prol da promoção da saúde assim como prevenção de doenças ${ }^{1}$.

No Brasil a Portaria n.o 2488/ 2011 aprova a Política Nacional de Atenção Básica, ajustando e retificando as condutas aplicadas para Estratégia de Saúde da Família (ESF) assim como para o Programa de Agentes Comunitários de Saúde (PACS) ${ }^{3}$.

Sua principal característica se dá pelo fato da observação do indivíduo pela sua singularidade e por considerar o ambiente sociocultural no qual está inserido. Tem seu atendimento e divisão de equipes organizados de acordo com área de abrangência a fim de estabelecer um vínculo mais estreito com a população adstrita e sua equipe multiprofissional de saúde correspondente ${ }^{4,5}$.

A fim de favorecer a afinidade entre a população atendida e equipe de saúde, há a figura do Agente Comunitário de Saúde (ACS) que, geralmente, faz parte da comunidade atendida na Unidade Básica de Saúde (UBS) a qual está alocado, o que possibilita o conhecimento das enfermidades e dores daquela comunidade, assim como proporciona facilidade ao acesso a esses indivíduos além de trazer a luz da equipe casos que necessitam de atenção ${ }^{4,6}$.

Em 11 de março de 2020 a OMS decretou Pandemia da COVID-19, doença causada pelo vírus Sars-CoV-2, levando o mundo a adotar medidas de precaução de contato e em 16 de março, o Governo do Estado do Rio de Janeiro, reconhecendo o cenário vivido, estabeleceu situação de emergência e decretou medidas para favorecer o distanciamento social a fim de reduzir os índices de contágio e, consequentemente, diminuir o número de $\operatorname{casos}^{4,7}$.

A Prefeitura do Rio de Janeiro, em consonância com o Governo Estadual, também adotou medidas que visaram a desaceleração da propagação da COVID-19. Entre essas medidas, estava a reorganização dos atendimentos prestados às UBS, que continuaram sendo a porta de entrada para o Sistema Único de Saúde (SUS), mas dessa vez o foco de atendimento estava voltado para indivíduos que apresentassem sintomas de síndrome gripal, sendo tratados como casos suspeitos da doença causada pelo novo coronavírus. Com isso os atendimentos regulares prestados por essas unidades tiveram que ser repensados e reestruturados.

Nesse cenário houve a implementação de uma prática utilizada por outros países e por empresas de planos de saúde no Brasil para acompanhamento da evolução de casos de doentes crônicos e agudos, o telemonitoramento ${ }^{5}$.

Esse tipo de acompanhamento consiste em verificar o andamento do tratamento estabelecido ao doente, avaliar seu quadro de saúde atual e classificar melhora ou piora através de contatos por chamadas telefônicas ou de vídeo $^{3,8,9}$.

Nesse contexto, o objetivo desse estudo foi avaliar a efetividade dessa prática de acompanhamento para os pacientes em tratamento de tuberculose, segundo a visão do enfermeiro, uma vez que práticas utilizadas para potencializar a adesão a essa terapêutica, como o Tratamento Diretamente Observado (TDO), por exemplo.

\section{Metodologia}

O estudo se trata de uma pesquisa de campo com abordagem qualiquantitativa realizada em uma Unidade de ESF localizada na Zona Norte do Município do Rio de Janeiro e a coleta de dados se deu entre os meses de setembro a novembro de 2020.

A análise de conteúdo de Bardin foi a metodologia escolhida para categorização e agrupamento dos dados coletados através do instrumento utilizado para realização da pesquisa. Houve o seguimento das etapas de pré-análise, exploração do material e tratamento e interpretação dos dados possibilitando a identificação das seguintes categorias: telemonitoramento como facilitador de criação/manutenção de vínculo entre a equipe e o paciente, cautela no uso de uma ferramenta que não possibilita o contato direto com o paciente, resistência para a aplicabilidade do telemonitoramento, o uso do telemonitoramento para alcance de localidades de difícil acesso e interferência da pandemia sobre o tratamento dos pacientes em curso de terapêutica de $\mathrm{TB}^{10}$.

A unidade utilizada como base para este estudo conta com 13 equipes da ESF, compostas por 01 enfermeiro, 01 médico e 04 ACSs por equipe. Também há a presença de residentes de Enfermagem e Medicina que realizam o atendimento à população em conjunto com os outros profissionais.

A amostra do estudo foi composta por 10 enfermeiros, entre eles os enfermeiros a frente da equipe (05) e residentes em seu segundo ano de residência (R2) (05), alocados nesta unidade. Todos os que aceitaram participar da pesquisa assinaram o Termo de Consentimento Livre e Esclarecido, além de terem obtido explicações acerca dos objetivos do estudo e da confidencialidade dos dados. Uma parcela das respostas obtidas, $40 \%$, se deu através de entrevista semiestruturada previamente agendada a fim de 
que não houvesse interrupção no fluxo de atendimento da Unidade. Devido ao período utilizado para coleta de dados, foi necessário a aplicação do instrumento através de Google Forms, já que houve o relaxamento das medidas de isolamento e a demanda de atendimento na unidade foi se tornando maior. As respostas que foram obtidas eletronicamente corresponderam a $60 \%$ do total.

Das 13 equipes que compõem a unidade, 03 estavam sem enfermeiros atuantes devido a licença médica ou falta de profissionais contratados. Haviam 10 enfermeiros a frente de equipes e 05 R2 atuantes na unidade, mas destes 15, 05 não estavam elegíveis para participação segundo os critérios de exclusão estabelecidos para este estudo. Os critérios de inclusão consistiram em: enfermeiros atuantes na unidade objeto do estudo no período de outubro a dezembro de 2020 e enfermeiros residentes que estejam em seu segundo ano de residência profissional além de enfermeiros que estejam atuantes na unidade a ser pesquisada pelo tempo mínimo de 6 meses anteriores à pandemia. Esses critérios visaram selecionar profissionais que conhecessem o perfil dos pacientes em tratamento de TB de sua equipe no período fora da Pandemia, pois assim seriam capazes de observar diferenças, mesmo que sutis, no que tange a adesão ao tratamento e frequência quanto ao comparecimento nas consultas e realização dos exames para controle.

Os aspectos éticos foram atendidos com as normas do Comitê de Ética e Pesquisa (CEP) da Secretaria Municipal de
Saúde do Rio de Janeiro (SMS/RJ) obtendo o parecer favorável (Parecer n.o 4.249.184) para dar prosseguimento com a pesquisa, sob o ofício CAAE no 36121520.9.0000.5279, cumprindo assim as exigências estabelecidas pelo Conselho Nacional de Saúde, na Resolução n.ㅇ 466/12. A execução ocorreu somente após a aprovação do CEP. A fim de proporcionar a privacidade, sigilo e anonimato dos participantes, foi registrado no verso dos formulários a inicial da profissão e um número sequencial de acordo com a realização das entrevistas, para as respostas obtidas de maneira eletrônica, não houve registro de dados capazes de identificar os indivíduos responsáveis porcada resposta.

\section{Resultados e Discussão}

No Quadro 1 podemos identificar a característica sociodemográfica dos profissionais de enfermagem que participaram do estudo e é possível observar o tempo de trabalho na ESF, idade e especialização. Através dos dados coletados é possível observar que essa equipe de enfermagem é composta por trabalhadores do sexo feminino, com idade média de 29,3 anos e com tempo de trabalho médio na ESF de 3 anos e 9 meses e com especialização em Saúde da família, em andamento para o caso dos residentes, e em $30 \%$ possuem também especialização em outras áreas da Enfermagem.

Quadro 1. Caracterização sociodemográfica da amostra ( $n=10)$. Rio de Janeiro, RJ, Brasil, 2020

\begin{tabular}{|c|c|c|c|c|}
\hline \multicolumn{5}{|c|}{ CARACTERIZAÇÃO SOCIODEMOGRÁFICA } \\
\hline Profissionais & Idade & Tempo na Estratégia & \multicolumn{2}{|l|}{ Especialização } \\
\hline Enf. 1 & 28 anos & 3 anos & \multicolumn{2}{|c|}{$\begin{array}{l}\text { Family Health and Medical and Surgical } \\
\text { Clinic }\end{array}$} \\
\hline Enf. 2 & 26 anos & 3 anos & \multicolumn{2}{|c|}{ Saúde da Família e Obstetrícia } \\
\hline Enf. 3 & 26 anos & 2 anos e 7 meses & \multicolumn{2}{|l|}{ Saúde da Família } \\
\hline Enf. 4 & 43 anos & 10 anos & \multicolumn{2}{|c|}{ Saúde da Família e Enfermagem do Trabalho } \\
\hline Enf. 5 & 32 anos & 4 anos & \multicolumn{2}{|l|}{ Saúde da Família } \\
\hline R2.1 & 24 anos & 1 ano e 7 meses & $\begin{array}{ll}\begin{array}{l}\text { Saúde } \\
\text { (em andamento) }\end{array} & \text { da }\end{array}$ & Família \\
\hline R2.2 & 25 anos & 1 ano e 7 meses & $\begin{array}{ll}\text { Saúde } & \text { da } \\
\text { (em andamento) }\end{array}$ & Família \\
\hline $\mathbf{R 2 . 3}$ & 25 anos & 1 ano e 8 meses & $\begin{array}{ll}\text { Saúde } & \text { da } \\
\text { (em andamento) }\end{array}$ & Família \\
\hline R2.4 & 30 anos & 1 ano e 8 meses & $\begin{array}{ll}\text { Saúde } & \text { da } \\
\text { (em andamento) }\end{array}$ & Família \\
\hline R2.5 & 34 anos & 1 ano e 8 meses & $\begin{array}{ll}\text { Saúde } & \text { da } \\
\text { (em andamento) }\end{array}$ & Família \\
\hline
\end{tabular}

Através de intensa leitura do material colhido e identificação de semelhanças nas falas de cada profissional entrevistado foi possível identificar cinco categorias capazes de conter as informações sobre a relação do telemonitoramento junto aos pacientes em curso de tratamento de TB e é possível notar que houve percepções distintas dos profissionais sobre essa prática adotada pelo SUS na tentativa de adaptação a Pandemia vivida ${ }^{8}$, embora hajam semelhanças quanto a faixa etária e especiaalização dos profissionais entrevistados, conforme descrito no Quadro 1. 
Telemonitoramento como facilitador de criação/manutenção de vínculo entre a equipe e o paciente

A estrutura estabelecida para proporcionar o atendimento à população junto as ESF têm como um dos alicerces o vínculo entre as equipes de saúde e os cidadãos vinculados às suas microáreas e a solidez dessa relação de confiança $^{6}$ pode significar a diferença entre o sucesso ou fracasso do tratamento. Dito isso pode-se mensurar a importância de uma ferramenta capaz de manter ou criar essa relação mesmo em tempos de distanciamento social devido a Pandemia de COVID-19 ${ }^{11}$.

"[...] a maioria gosto, é principalmente idosos que eles são sozinhos então acabava que viravam consulta que eles queriam conversar da vida né, acabava virando até uma consulta de psicologia, assim. É mais foi bem proveitoso pra eles, ninguém, não teve nenhuma negativa não" (Enf.1).

"[...] se sentiram acolhidos e assistidos nesse período de isolamento" (R2.2).

\section{Cautela no uso de uma ferramenta que não possibilita o contato direto com o paciente}

Para que o cuidado integral, centrado no paciente, seja desenvolvido de maneira efetiva, a ESF propõe equipes multiprofissionais integradas assim como ações complementares entre si, ampliando a capacidade de alcance do cuidado prestado ${ }^{9}$. Parte desse alcance consiste em informações que são analisadas mesmo que o paciente não verbalize e a falta dessa observância pode interferir no tratamento.

"[...] muita coisa a gente vê, nem tudo eles falam [...] acho que olhar o semblante do paciente, observar seu autocuidado de perto $e$, às vezes, até o jeito dele falar, interfere nas decisões que a gente toma para dar um cuidado de maior qualidade [...] só isso de telefone não alcança isso" (Enf.5).

"[...] olha, eu acredito que em partes assim, porque eu acho o contato com o paciente, é mais eficiente do que só ficar ligando, eu acho que acabo distanciando um pouco do paciente. Acho que talvez, assim, a renovação de receita algo mais pontual dá para ser telemonitorado. Agora o restante não tem como" (Enf. 2).

"[...] acredito que grande parte se sinta feliz por estar sendo acessado, mas muitos continuam tendo a necessidade de acompanhamento presencial" (R2.1).

\section{Resistência para a aplicabilidade do telemonitoramento}

Embora ainda não fosse prática concreta utilizada em larga escala pelo SUS, outros países e empresas privadas de planos de saúde no Brasil identificarambenefícios como: efetividade em saúde preventiva, consequente redução de uso de serviços de urgência/ emergência, aumento de expectativa de vida e também é considerado como importante ferramenta para o enfrentamento de crescimento de custos causado pelo tratamento de Doenças Crônicas Não Transmissíveis (DCNT) ${ }^{11,12}$. Ainda assim, a falta de estudos que comprovem a efetividade dessa prática pode ser a razão por ela gerar resistência dos profissionais de saúde em utilizá-la.

"[...] a presença de um profissional, proporciona um olhar ampliado para o paciente, assim como para o contexto em que ele está inserido" (R2.3).

"[...] acredito que é importante estar em contato direto com o paciente para entender melhor sua situação de saúde [...]" (R2.4).

\section{O uso do telemonitoramento para alcance de localidades de difícil acesso}

É sabido que esse tipo de acompanhamento proporciona o atendimento em saúde em locais com estrutura deficitária ou de difícil acesso ${ }^{10}$ e isso poderia significar aumento da adesão populacional ao SUS e possibilitar não só o tratamento de doenças que já possuem, assim como tornar-se ferramenta para a promoção a saúde e prevenção de doenças ${ }^{12}$.

"[...] acredito ser uma ferramenta importantíssima. Porque onde a gente não chega fisicamente, a gente pode chegar de uma outra forma. Acho de extrema importância" (Enf.4).

"[...] teve vantagem porque a gente acabou que tipo conseguiu falar com esses pacientes e saber quem não tava em, é com a rotina né, tava desacompanhado, então vinha aqui na unidade e a gente buscava" (Enf.3).

\section{Interferência da pandemia sobre o tratamento dos} pacientes em curso de terapêutica de TB

A adesão e manutenção do tratamento é um dos desafios traçados entre o serviço de assistência à saúde e a tuberculose. Consiste em um tratamento multidimensional e fatores como o conhecimento da doença, compreensão do tratamento tal qual a relação entre o sistema de saúde, seus profissionais e o paciente é essencial para que a terapêutica seja efetiva e que esse paciente chegue à alta através da cura. Por ser um tratamento extenso e a principal ferramenta para que fosse garantido o uso diário do medicamento através do TDO teve que ser interrompido devido a Pandemia, ouviu- se na fala desses profissionais que houve um grande impacto no tratamento desses pacientes ${ }^{10-}$ 13 .

"[...] em alguns casos interferiu. Quando o ACS ia até a casa do paciente entregar os medicamentos da semana, percebia que ainda tinham medicações que deveriam ter sido utilizadas na semana anterior. Com o TDO, era possivel auxiliar aqueles com maior dificuldade" (R2.4).

"[...] com certeza interferiu, assim, é nós tivemos pacientes que deram abandono [...] justamente por esse fato e por não ter conseguido fazer as doses supervisionadas, a gente não tinha certeza se o paciente estava tomando ou não [...]" (Enf.4). 
0 tratamento diretamente observado para tuberculose pode ser substituído e, ainda assim, obter sucesso no tratamento? Côrtes EMP, Silva WBH, Ferreira MA, Mattos CM, Machado PRF, Lima TA, Marta CB, Silva Junior MD

\section{Considerações Finais}

Através desse estudo foi possível perceber que a pandemia, como esperado, afetou a adesão do tratamento de TB. Embora não tenha sido relatado abandono propriamente dito, houveram situações em que pode-se observar uso inadequado da terapia medicamentosa prescrita, o que pode gerar consequências como o desenvolvimento de tuberculose multidroga resistente além de interferir, diretamente, sobre o tempo necessário para conclusão da terapêutica estabelecida para esses pacientes.

Sobre o telemonitoramento é possível inferir que, embora com ressalvas, foi uma prática eficaz para o acompanhamento dos pacientes em tratamento de TB, pois proporcionou o fortalecimento do vínculo entre o paciente e o serviço de saúde e favoreceu o acolhimento desses pacientes que, por serem acompanhados de maneira mais frequente pela clínica, se sentiriam "desassistidos" devido a diminuição da frequência do contato pessoal.

Podemos citar como principal ponto falho do telemonitoramento a falta de possibilidade de ver o paciente, o que nos causa a perda da observação de sinais e/ou sintomas que não são referidos pelo por ele, o que gera perda da assistência voltada para o indivíduo de maneira completa.

Para futuras pesquisas foi identificada a necessidade de verificar os índices de abandono do tratamento de TB durante o período pandêmico e analisar também os números no que se refere ao tratamento de TB multidroga resistente visto que esses são os principais riscos dos pacientes em tratamento de TB.

\section{Referências}

1. Organização Mundial de Saúde (OMS). The End TB Strategy [Internet]. OMS, 2015 [acesso em 2020 dez 02]. Disponível em: https://www.who.int/tb/End_TB_brochure.pdf?ua=1

2. Ministério da Saúde (BR). Manual de recomendações para o controle da tuberculose no Brasil. Brasília (DF): MS; 2019.

3. Linhares SRS, Paz EPA. A vivência do tratamento de tuberculose em unidades de Saúde da Família. Escola Anna Nery [Internet] 2020 [acesso em 2020 dez 02]24(2):1-7. Disponível em: https://www.scielo.br/scielo.php?pid=S1414$81452020000200216 \&$ script $=$ sci_arttext

4. Mussi FC, Palmeira CS, Silva RM, Costa ALS. Telenfermagem: contribuições para o cuidado em saúde e a promoção do conforto. Rev. Cient. Sena Aires [Internet]. 2018 [acesso em 2020 dez 03];7(2):76-9. Disponível em: http://revistafacesa.senaaires.com.br/index.php/revisa/article/viewFile/306/216

5. Kahl C, Meirelles BHS, Cunha KS, Bernardo MS, Erdmann AL. Contribuições da prática clínica do enfermeiro para o cuidado na Atenção Primária. Rev Bras Enferm. 2019;72(2):354-9. http://dx.doi.org/10.1590/0034-7167-2018-0348

6. Faria RM. A territorialização da Atenção Básica à Saúde do Sistema Único de Saúde do Brasil. Ciência saúde coletiva [Internet]. 2020 nov [acesso em $2020 \mathrm{dez}$ 05];25(11). Disponível em: https://www.scielo.br/scielo.php?script=sci_arttext\&pid=S1413$81232020001104521 \&$ lang=pt

7. Daumas RP, Silva GA, Tasca R, et al. O papel da atenção primária na rede de atenção à saúde no Brasil: limites e possibilidades no enfrentamento da COVID-19. Cad. Saúde Pública [Internet]. 2020 jun [acesso em 2020 dez 01];36(6). Disponível em: https://www.scielo.br/scielo.php?script=sci_arttext\&pid=S0102-311X2020000600503\&tlng=pt

8. Sousa GJB, Garces TS, Pereira MLD, et al. Temporal pattern of tuberculosis cure, mortality, and treatment abandonment in Brazilian capitals. Rev Latin-Am Enferm [Internet]. 2019 ago [acesso em 2020 set 22];27:e3218. Disponível em: https://www.scielo.br/scielo.php?script=sci_arttext\&pid=S0104-11692019000100399.

9. Caetano R, Silva AB, Guedes ACCM, et al. Desafios e oportunidades para telessaúde em tempos da pandemia pela COVID-19: uma reflexão sobre os espaços e iniciativas no contexto brasileiro. Cadernos de Saúde Pública [Internet]. 2020 mai [acesso em 2020 set 25];36(5). Disponível em: https://www.scielo.br/scielo.php?script=sci_arttext\&pid=S0102-311X2020000503001

10. Bardin L. Análise de conteúdo. Lisboa: Edições; 2016.

11. Paula AC, Maldonado JMSV, Gardelha CAG. Telemonitoramento e a dinâmica empresarial em saúde: desafios e oportunidades para o SUS. Rev Saúde Pública [Internet] 2020 [acesso em 29 nov 2020];54:65. Disponível em: https://www.scielosp.org/pdf/csp/2016.v32suppl2/e00150115/pt

12. Silva WBH, Côrtes EMP, Lima TA, et al. Influência da Pandemia da Covid-19 nos índices glicêmicos dos pacientes diabéticos. Research, Society and Development [Internet]. 2020 nov [acesso 2020 nov 29];9(11). Disponível em: https://rsdjournal.org/index.php/rsd/article/download/10427/9217/141311

13. Orlandi GM, Pereira EG, Biagolini REM, et al. Incentivos sociais na adesão ao tratamento da tuberculose. Rev Bras Enferm [Internet]. 2019 [acesso em $2020 \mathrm{dez}$ 02];72(5):1247-1253. Disponível em: https://www.scielo.br/scielo.php?script=sci_abstract\&pid=S0034- 71672019000501182\&lng=en\&nrm=iso\&tlng=pt

14. Marta CB, Silva WBH, Côrtes EMP, Machado TO, Francisco MTR, Silva PO, et al. Telemonitoramento: análise da percepção dos acadêmicos de enfermagem frente à pandemia da COVID-19. Glob Acad Nurs. 2020;1(3):e52. https://doi.org/10.5935/26755602.20200052 